\section{Mini-Review}

\author{
Correspondence \\ Kai M. Thormann \\ thormann@mpi-marburg.mpg.de
}

\title{
Tuning the flagellar motor
}

\author{
Kai M. Thormann and Anja Paulick \\ Department of Ecophysiology, Max-Planck-Institut für Terrestrische Mikrobiologie, Marburg, \\ Germany
}

\begin{abstract}
Many bacteria are motile by means of flagella, semi-rigid helical filaments rotated at the filament's base and energized by proton or sodium-ion gradients. Torque is created between the two major components of the flagellar motor: the rotating switch complex and the cell-wall-associated stators, which are arranged in a dynamic ring-like structure. Being motile provides a survival advantage to many bacteria, and thus the flagellar motor should work optimally under a wide range of environmental conditions. Recent studies have demonstrated that numerous species possess a single flagellar system but have two or more individual stator systems that contribute differentially to flagellar rotation. This review describes recent findings on rotor-stator interactions, on the role of different stators, and on how stator selection could be regulated. An emerging model suggests that bacterial flagellar motors are dynamic and can be tuned by stator swapping in response to different environmental conditions.
\end{abstract}

\section{Introduction}

The perception that many bacteria are motile dates back to the very beginning of microscopy, marked by the optical devices built by Antonie van Leeuwenhoek. Fascinated by the different types of motion by the 'little living animalcules', he envisioned the cells to possess tiny 'paws' to enable the 'strong and swift motion' he observed (Dobell, 1960). Now, some 330 years later, it is clear that the tiny paws are, in fact, bacterial flagella, rotating helical proteinaceous filaments extending from the cell's surface that act as propellers to drive the cells through liquid environments or across surfaces. Flagella-mediated swimming motility certainly represents a widespread and a very effective means of active locomotion among bacteria. With a chemotaxis-navigated motility system, a bacterium gains the ability to actively leave non-favourable environments to seek out more beneficial conditions, resulting in a significant survival advantage (Armitage, 1999). A functional flagellar system is required for community formation in many species, and the flagellum is also implicated in measuring environmental conditions such as viscosity or wetness (McCarter et al., 1988; Wang et al., 2005). Thus, for many species, flagella-mediated motility is a significant, or even crucial, factor in successful propagation. It is, therefore, important that the flagellar system functions under a wide range of environmental conditions.

This review focuses on species that use different motor configurations to tune the performance of the flagella according to the environmental conditions. Recent findings on the nature of rotor-stator interactions and dynamics in the flagellar motor offer novel and intriguing perspectives on how motor functions may be modulated. In this context, a model will be developed and discussed.

\section{The flagellar motor}

The first of the three major components of the bacterial flagellar system (Fig. 1) is the prominent filament. The second, the basal body, anchors the flagellum to the cell envelope and is engaged in rotation of the filament. The third major part, referred to as the hook, serves as a universal joint that connects the filament to the basal body. The flagellar structure is built from inside out and requires some 50 genes, whose expression is regulated in a hierarchy of several levels (Macnab, 2003; McCarter, 2006). The motor that drives flagellar rotation is an intricate molecular nanomachine fuelled by transmembrane ion gradients. Some flagellar motors work bidirectionally and allow alternation between clockwise and counterclockwise rotation; others function exclusively in a unidirectional manner. Thus far, two coupling ions, $\mathrm{H}^{+}$and $\mathrm{Na}^{+}$, have been described as energy sources.

Due to its size, complexity, and localization in the membrane, it has so far been impossible to obtain atomic structures of a complete flagellar motor system. However, a combination of partial X-ray structures of motor proteins, site-directed mutagenesis, electron microscopic imaging and recent cryo-electron tomography imaging has led to rather detailed models (Liu et al., 2009; Minamino et al., 2008; Thomas et al., 2006). Although some differences, in particular ones regarding the complex protein structures of the basal body, occur among bacterial species, some general characteristics appear to be shared by all flagellar systems. All require two major components for the conversion of ion flux into the torque that drives rotation of the flagellum. One is the rotor-mounted switch complex (C ring), which is composed of multiple copies of the FliG, FliM and FliN proteins (Fig. 1). Interaction between 


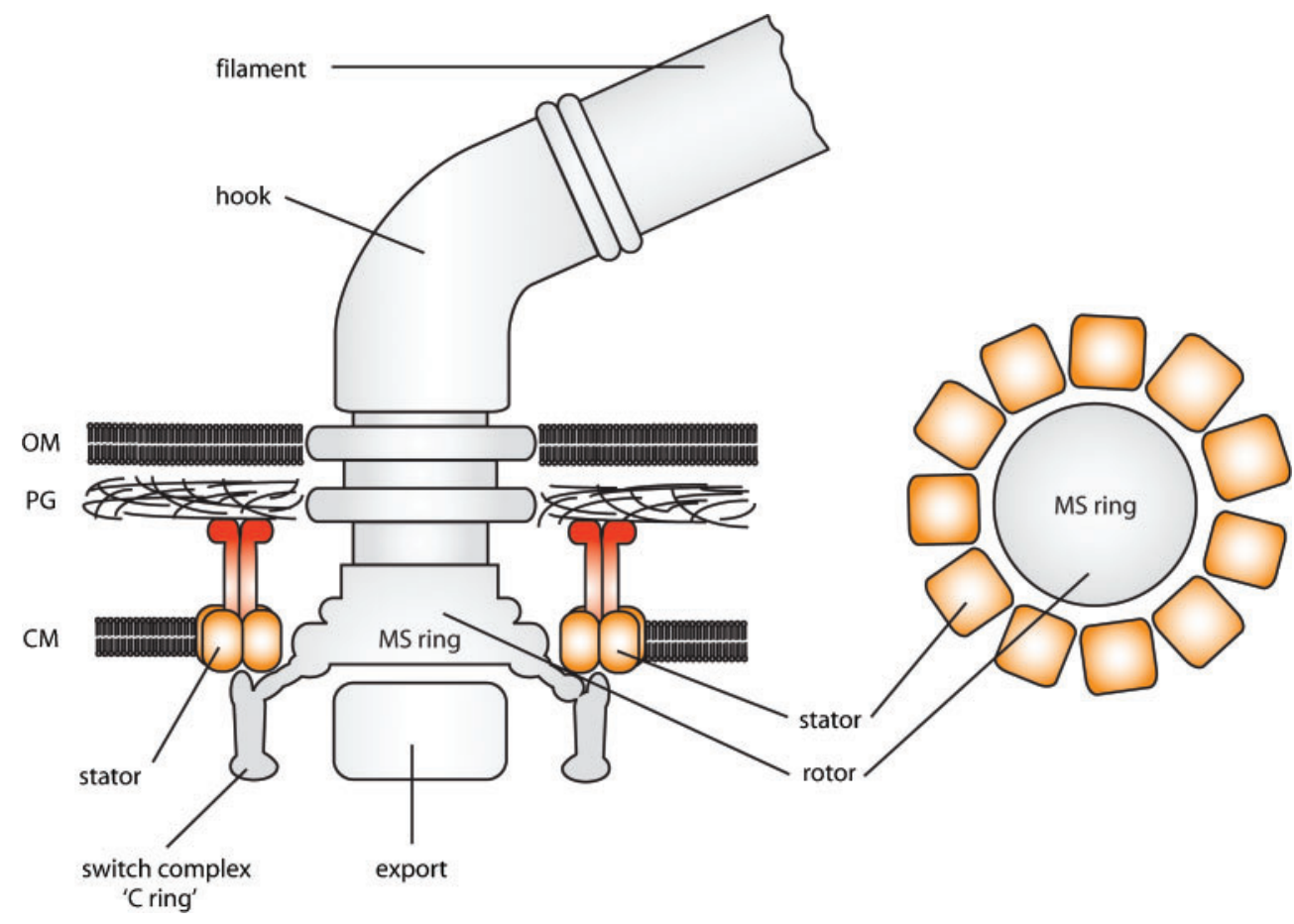

Fig. 1. The main components of the flagellar motor. Left: simplified model of the flagellar basal body-motor complex showing the main components, including the hook and filament. Right: schematic top view of the motor-stator complex. The stator complexes are assumed to surround the MS-ring structure. In E. coli, about 11 stator complexes can synchronously be engaged in rotor-stator interactions. OM, outer membrane; PG, peptidoglycan; CM, cytoplasmic membrane.

the switch complex and the chemosensory system allows modulation of the motor activity, for example by switching the direction of rotation, as occurs in Escherichia coli. The second major motor component is the stator system, which consists of several membrane-embedded stator complexes that determine the specificity for the coupling ion. The exact mechanism of torque generation still remains obscure.

Numerous studies have been devoted to unravelling the structure, assembly and function of the flagellar motor, and the subject has been recently reviewed (Kojima \& Blair, 2004a; Berg, 2003; Minamino et al., 2008; Sowa \& Berry, 2008; Kojima et al., 2008).

\section{Rotor-stator interactions}

So far, isolation of a complete basal complex including the stator units has not been successful. Based on earlier electron micrographs of freeze-fractured cells that revealed the presence of membrane-embedded particle rings, it is assumed that the stator complexes form a ring surrounding the MS ring above the switch complex. This was recently confirmed by cryo-electron tomography studies on intact flagellar motors of Borrelia burgdorferi (Khan et al., 1988, 1992; Coulton \& Murray, 1978; Minamino et al., 2008; Thomas et al., 2006; Liu et al., 2009) (Fig. 1). The number of stator units depends on the bacterial species; in E. coli,
11 or 12 stator complexes can be synchronously involved in flagellar rotation (Reid et al., 2006; Leake et al., 2006). Each stator complex is composed of two different subunits, commonly denoted as MotA and MotB (in the protondependent E. coli stator) or PomA and PomB (in the $\mathrm{Na}^{+}$-dependent stator of Vibrio species), which are found in a $4: 2$ stoichiometry (Braun et al., 2004; Kojima \& Blair, 2004b; Sato \& Homma, 2000). It is postulated that two A-subunits and one B-subunit form a single ion channel, so that each stator complex would contain two channels (Braun et al., 2004). The B-subunit has a single transmembrane domain, and a peptidoglycan-binding motif located at the C-terminus of MotB/PomB tethers the complex to the cell wall and is essential for stator function. The A-subunits have four transmembrane domains; a cytoplasmic loop connecting the transmembrane domains 2 and 3 is thought to interact with FliG in the switch complex (Lloyd et al., 1996; Zhou et al., 1998; Yorimitsu et al., 2003) (Fig. 2).

Mutational studies have identified several charged residues in the cytoplasmic segment of MotA and the C-terminus of FliG in the proton-driven E. coli motor that are involved in electrostatic interactions between the two proteins. These interactions were shown to be rather robust, and introduction of mutations leading to charge reversal in one, or charge neutralization in two, of these residues was required to strongly affect motor function (Lloyd \& Blair, 
(a)

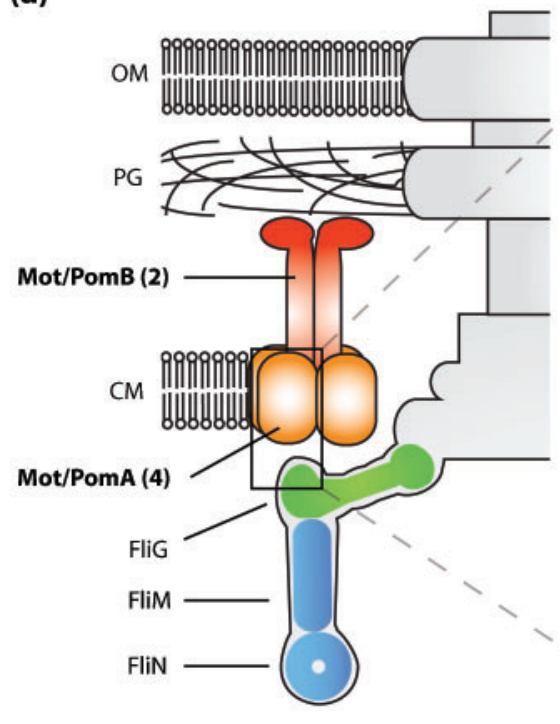

(b)

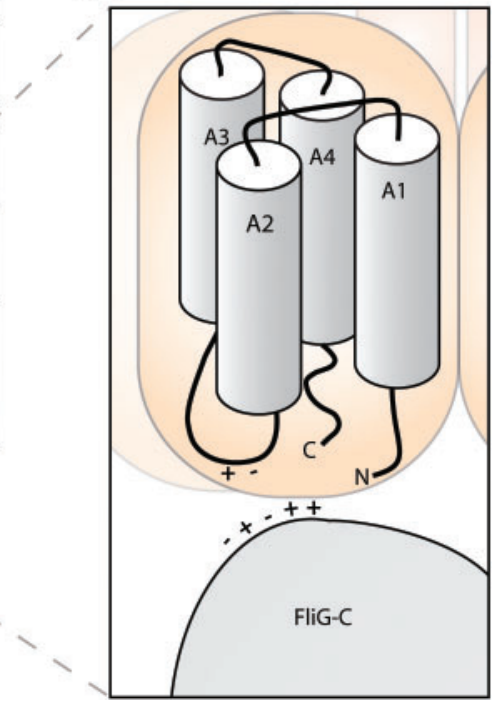

Fig. 2. Rotor-stator interactions. (a) The stators are assumed to interact with the switch complex, which is composed of the FliG, FliM and FliN proteins. Each stator complex consists of four A and two B subunits. (b) One MotA subunit has four transmembrane domains (A1-A4). Charged amino acid residues located in the $\mathrm{C}$-terminal domain of FliG and the cytoplasmic loop connecting A2 and A3 are assumed to interact electrostatically.
1997; Zhou \& Blair, 1997; Zhou et al., 1998). A comparative study of chimeric motors using the Vibrio alginolyticus stator and/or rotor components in an E. coli background strongly indicated that PomA and FliG interact in a similar manner to E. coli MotA and FliG. However, the interactions of PomA and FliG were demonstrated to be less sensitive to mutations in the conserved charged residues in Vibrio species (Yorimitsu et al., 2003, 2002). This elevated robustness of PomA and FliG interactions in the $\mathrm{Na}^{+}$-driven motor was partly attributed to several additional charged residues in PomA, but it is assumed that other factors play a more important role (Yakushi et al., 2006). Such factors might be auxiliary proteins such as MotX and MotY, which have been shown to be required for stator recruitment to the flagellar motor and motor function in Vibrio and other species (Koerdt et al., 2009; Terashima et al., 2006). In addition, it was demonstrated that FliG can functionally interact with 'foreign' stators. Expression of PomA and a modified PomB variant in $E$. coli supported flagellar rotation in a $\mathrm{Na}^{+}$-dependent fashion (Asai et al., 2003; Sowa et al., 2005). Conversely, the E. coli MotAB stator supported proton-dependent function in motors of Vibrio species (Asai et al., 2003; Gosink \& Häse, 2000). Taken together, these studies show that functional interactions between rotors and stators of different species may be rather flexible and that the stators determine the activity of the flagellar motor. This also means that a flagellar motor might be able to function with different sets of stators. In fact, this situation occurs in several bacterial species.

\section{Multiple stator systems provide different motor functions}

Most flagellated bacterial species possess one or more distinct flagellar systems that are driven by individual motor/stator partners to provide swimming and/or swarm- ing motility. In contrast, several species have been described in which the number of distinct putative stators exceeds that of the flagellar motor systems. Do these organisms use different rotor-stator configurations to modify the motor function? Four dual-stator systems that have been characterized in more detail strongly suggest that this is the case.

Pseudomonas aeruginosa PAO1 is motile by virtue of a single polar flagellum and has two distinct protondependent stator systems, MotAB and MotCD. Both stators can individually support swimming in liquid environments and swarming across surfaces, albeit at different efficiencies. Although the MotAB stator outperforms MotCD in liquid environments, MotCD supports swimming more effectively under conditions of elevated viscosity or swarming across surfaces (Doyle et al., 2004; Toutain et al., 2005). The presence of both stators is required for effective colonization of abiotic surfaces (Toutain et al., 2007).

Aeromonas hydrophila possesses two flagellar systems, one of which is a single polar flagellum. The second system consists of a set of lateral flagella (Shimada et al., 1985). Both systems depend on individual stator systems (PomAB and LafTU, repectively). Recently, a third stator system, Pom $A_{2} B_{2}$, has been identified, and it has been shown that the two distinct $\mathrm{Na}^{+}$-dependent stators, PomAB and Pom $A_{2} B_{2}$, are involved in rotation of the polar flagellum. Mutant analysis suggests that the $\operatorname{PomA}_{2} \mathrm{~B}_{2}$ system supports faster swimming under conditions of low sodium (Wilhelms et al., 2009).

In contrast to the above-mentioned $P$. aeruginosa and A. hydrophila, which harbour two stator complex systems that depend on the same coupling ion, the dual stator systems of Bacillus subtilis and Shewanella oneidensis MR-1 consist of one $\mathrm{H}^{+}$- and one $\mathrm{Na}^{+}$-dependent stator. In 
B. subtilis, the $\mathrm{H}^{+}$-dependent MotAB stator is dominant for the function of the peritrichous flagellar system. However, the $\mathrm{Na}^{+}$-powered MotPS stator contributes to swimming speeds at elevated $\mathrm{pH}$ and $\mathrm{Na}^{+}$concentrations and to locomotion at higher viscosity (Ito et al., 2004, 2005). In $S$. oneidensis MR-1, the single polar flagellum is predominantly powered by the $\mathrm{Na}^{+}$-dependent PomAB system, but it is supported by the proton-dependent MotAB stator at low sodium concentrations (Paulick et al., 2009).

The studies on the flagellar motors of these four organisms clearly demonstrate that individual stator systems support the motor function differentially (Table 1). It is easily conceivable that a switch in the motor-driving coupling ion, as occurs in B. subtilis and S. oneidensis MR-1, would change the properties of the motor and expand the range of flagellar functions, for example, in environments with rapidly changing $\mathrm{pH}$ and $\mathrm{Na}^{+}$concentrations. However, as the studies on $P$. aeruginosa and $A$. hydrophila demonstrate, stators that depend on the same coupling ion can also change the properties of the flagellar motor with regard to speed or performance under load (Doyle et al., 2004; Toutain et al., 2005; Wilhelms et al., 2009). Thus, different sets of stators might enable their corresponding host species to fine-tune the motor function according to environmental conditions. As opposed to a second complete flagellar system, a dual/multiple stator system represents a comparatively inexpensive means to upgrade the bacterial motility machine. In addition, a two-gene operon is more likely to evolve as a paralogue within the same genome or could be easily acquired by horizontal gene transfer. For instance, genome analysis in S. oneidensis MR-1 strongly suggests that the proton-dependent MotAB stator system has been rather recently acquired from a different species in the process of a long-term transition from a marine to a freshwater environment (Paulick et al., 2009).

How common are multiple stator systems? A systematic analysis of the available bacterial genomic data identified more than 65 species that possess a single flagellar motor system with two or more putative stators. These species are widely distributed among different Gram-positive and Gram-negative genera (Table 2). Although most have dual stator systems along with a single flagellar motor system, the number of distinct potential stators for a single motor system can be as high as four, for example in some Desulfovibrio species. Thus, a dual or multiple stator drive appears to be surprisingly common, given the fact that our analysis only covered species with a single flagellar motor and did not include bacteria such as Aeromonas with a twomotors/three-stators ratio.

The major question arising is how regulation of stator selection occurs in these organisms. Do these species use

Table 1. Differential role of bacterial stator systems in flagellar function

\begin{tabular}{|c|c|c|c|c|c|c|}
\hline Species & $\begin{array}{l}\text { Flagellar } \\
\text { system }(s)\end{array}$ & Stator systems & $\begin{array}{l}\text { Energy source/ } \\
\text { coupling ion }\end{array}$ & $\begin{array}{c}\text { Ratio } \\
\text { flagella: stator }\end{array}$ & Function & Reference(s) \\
\hline \multirow[t]{4}{*}{$\begin{array}{l}\text { Aeromonas } \\
\text { hydrophila }\end{array}$} & $\begin{array}{l}\text { Single polar } \\
\text { flagellum }\end{array}$ & PomAB & smf & $2: 3$ & PomAB: swimming, swarming & Merino et al. (2006) \\
\hline & & $\operatorname{PomA}_{2} \mathrm{~B}_{2}$ & $\operatorname{smf}$ & & $\begin{array}{l}\text { Pom }_{2} B_{2} \text { : swimming at low } \\
\text { sodium (?) }\end{array}$ & Shimada et al. (1985) \\
\hline & & & & & Biofilm formation (?) & Wilhelms et al. (2009) \\
\hline & Lateral flagella & MotAB/LafTU & $\mathrm{pmf}$ & & LafTU: swimming, swarming & \\
\hline \multirow[t]{2}{*}{ Bacillus subtilis } & $\begin{array}{l}\text { Peritrichous } \\
\text { flagella }\end{array}$ & $\begin{array}{l}\text { MotAB } \\
\quad(\text { BSU13690/13680) }\end{array}$ & pmf & $1: 2$ & MotAB: swimming, swarming & Ito et al. $(2004,2005)$ \\
\hline & & $\begin{array}{l}\text { MotPS } \\
\text { (BSU29730/29720) }\end{array}$ & smf & & $\begin{array}{l}\text { MotPS: swimming at elevated } \\
\text { viscosity, } \mathrm{pH} \text { and } \mathrm{Na}^{+} \\
\text {concentrations, biofilm } \\
\text { formation }\end{array}$ & \\
\hline \multirow[t]{2}{*}{$\begin{array}{l}\text { Pseudomonas } \\
\text { aeruginosa } \mathrm{PAO} 1\end{array}$} & $\begin{array}{l}\text { Single polar } \\
\text { flagellum }\end{array}$ & $\begin{array}{l}\text { MotAB } \\
(\text { PA4954/4953) }\end{array}$ & $\mathrm{pmf}$ & $1: 2$ & MotAB: swimming in liquid & Doyle et al. (2004) \\
\hline & & $\begin{array}{l}\text { MotCD } \\
(\text { PA1460/1461) }\end{array}$ & $\mathrm{pmf}$ & & $\begin{array}{l}\text { MotCD: swimming at elevated } \\
\text { viscosity, swarming } \\
\text { Both: swimming, swarming, } \\
\text { biofilm formation }\end{array}$ & $\begin{array}{l}\text { Toutain et al. }(2005 \text {, } \\
\text { 2007) }\end{array}$ \\
\hline $\begin{array}{l}\text { Shewanella } \\
\text { oneidensis MR-1 }\end{array}$ & $\begin{array}{l}\text { Single polar } \\
\text { flagellum }\end{array}$ & $\begin{array}{l}\text { MotAB } \\
(\text { SO4287/4286) } \\
\text { PomAB } \\
(\text { SO1529/1530) }\end{array}$ & $\begin{array}{l}\mathrm{pmf} \\
\mathrm{smf}\end{array}$ & $1: 2$ & $\begin{array}{l}\text { MotAB: swimming at low } \\
\mathrm{Na}^{+} \text {concentrations } \\
\text { PomAB: swimming }\end{array}$ & Paulick et al. (2009) \\
\hline
\end{tabular}

${ }^{*}$ pmf, proton-motive force; smf, sodium-motive force. 
Table 2. Bacterial species with a single flagellar motor and multiple stator systems

\begin{tabular}{|c|c|}
\hline Species with a single putative motor system ${ }^{\star}$ & $\begin{array}{l}\text { No. of putative } \\
\text { stator systems } \dagger\end{array}$ \\
\hline \multicolumn{2}{|l|}{ Gram-negative bacteria } \\
\hline \multicolumn{2}{|l|}{ Proteobacteria } \\
\hline \multicolumn{2}{|l|}{ Alphaproteobacteria } \\
\hline Rhodospirillum rubrum ATCC 11170 & 2 \\
\hline Zymomonas mobilis subsp. mobilis & 2 \\
\hline \multicolumn{2}{|l|}{ Betaproteobacteria } \\
\hline Azoarcus sp. BH72 & 2 \\
\hline Burkholderia ambifaria & 2 \\
\hline Burkholderia cenocepacia $\ddagger$ & 2 \\
\hline Burkholderia glumae BGR1 & 3 \\
\hline Burkholderia multivorans ATCC 17616 & 2 \\
\hline Burkholderia phymatum STM815 & 2 \\
\hline Burkholderia phytofirmans PsJN & 2 \\
\hline Burkholderia sp. 383 & 3 \\
\hline Burkholderia vietnamiensis $\mathrm{G} 4$ & 2 \\
\hline Burkholderia xenovorans LB400 & 2 \\
\hline Dechloromonas aromatica $\mathrm{RCB}$ & 2 \\
\hline Herminiimonas arsenicoxydans & 2 \\
\hline Janthinobacterium sp. Marseille & 2 \\
\hline Methylobacillus flagellatus KT & 2 \\
\hline Nitrospira multiformis ATCC 25196 & 2 \\
\hline Thauera sp. MZ1T & 2 \\
\hline \multicolumn{2}{|l|}{ Gammaproteobacteria } \\
\hline Azotobacter vinelandii DJ & 2 \\
\hline Cellvibrio japonicus Ueda107 & 2 \\
\hline Chromohalobacter salexigens DSM 3043 & 2 \\
\hline Colwellia psychrerythraea $34 \mathrm{H}$ & 2 \\
\hline Halorhodospira halophila SL1 & 3 \\
\hline Legionella pneumophila $\ddagger$ & 2 \\
\hline Marinobacter aquaeolei VT8 & 4 \\
\hline Marinomonas sp. MWYL1 & 3 \\
\hline Pseudoalteromonas haloplanktis TAC125 & 2 \\
\hline Pseudomonas aeruginosa $\ddagger$ & 2 \\
\hline Pseudomonas entomophila $\ddagger$ & 2 \\
\hline Pseudomonas fluorescens $\ddagger$ & 2 \\
\hline Pseudomonas putida $\ddagger$ & 2 \\
\hline Pseudomonas stutzeri A1501 & 3 \\
\hline Pseudomonas syringae $\ddagger$ & 2 \\
\hline Saccharophagus degradans 2-40 & 2 \\
\hline Shewanella oneidensis MR-1 & 2 \\
\hline Stenotrophomonas maltophila $\ddagger$ & 2 \\
\hline Teredinibacter turnerae T7901 & 2 \\
\hline Thioalkalivibrio sp. HL-EbGR7 & 4 \\
\hline Thiomicrospira crunogena XCL-2 & 3 \\
\hline Vibrio fischeri $\ddagger$ & 2 \\
\hline Xanthomonas axonopodis pv. citri strain 306 & 2 \\
\hline Xanthomonas campestris pv. campestris $\ddagger$ & 2 \\
\hline Xanthomonas oryzae pv. oryzae $\ddagger$ & 2 \\
\hline \multicolumn{2}{|l|}{ Deltaproteobacteria } \\
\hline Bdellovibrio bacteriovorus HD100 & 3 \\
\hline Desulfobacterium autotrophicum HRM2 & 3 \\
\hline $\begin{array}{l}\text { Desulfovibrio desulfuricans subsp. desulfuricans } \\
\text { G20 }\end{array}$ & 2 \\
\hline Desulfovibrio magneticus RS-1 & 4 \\
\hline
\end{tabular}

Table 2. cont.

\begin{tabular}{|c|c|}
\hline Species with a single putative motor system ${ }^{\star}$ & $\begin{array}{l}\text { No. of putative } \\
\text { stator systems } \dagger\end{array}$ \\
\hline Desulfovibrio salexigens DSM 2638 & 4 \\
\hline Desulfovibrio vulgaris strain Miyazaki F & 4 \\
\hline Desulfovibrio vulgaris $\ddagger$ & 3 \\
\hline Lawsonia intracellularis PHE/MN1-00 & 2 \\
\hline \multicolumn{2}{|l|}{ Nitrospirae } \\
\hline Thermodesulfovibrio yellowstonii DSM 11247 & 2 \\
\hline \multicolumn{2}{|l|}{ Acidobacteria } \\
\hline 'Candidatus Koribacter versatili' Ellin 345s & 2 \\
\hline 'Candidatus Solibacter usitatus' Ellin 6076 & 3 \\
\hline \multicolumn{2}{|l|}{ Gram-positive bacteria } \\
\hline \multicolumn{2}{|l|}{ Firmicutes } \\
\hline Bacillus amyloliquefaciens FZB42 & 2 \\
\hline Bacillus anthracis $\ddagger$ & 2 \\
\hline Bacillus cereus $\ddagger$ & 2 \\
\hline Bacillus cytotoxicus NVH 391-98 & 2 \\
\hline Bacillus licheniformis ATCC 14580 & 2 \\
\hline Bacillus subtilis $\ddagger$ & 2 \\
\hline Bacillus thuringiensis $\ddagger$ & 2 \\
\hline Bacillus weihenstephanensis KBAB4 & 2 \\
\hline Oceanobacillus iheyensis HTE831 & 2 \\
\hline \multicolumn{2}{|l|}{ Clostridia } \\
\hline Clostridum acetobutylicum ATCC 824 & 2 \\
\hline Clostridium beijerinckii NCIMB 8052 & 3 \\
\hline Clostridium botulinum $\ddagger$ & 2 \\
\hline Desulfitobacterium hafniense Y51 & 2 \\
\hline Desulfotomaculum reducens MI-1 & 2 \\
\hline Moorella thermoacetica ATCC 25196 & 3 \\
\hline
\end{tabular}

${ }^{*}$ Motor systems were identified by sequence alignment of FliF of $S$. oneidensis MR-1 against fully sequenced bacterial genomes by BLASTP with an $E$-value cutoff of $<1 \times 10^{-10}$. Candidate genes were then tested for their neighbouring sequence. Only candidate species with a single fliF in the vicinity of other flagellar related genes were considered.

$\dagger$ Putative stator systems were identified by sequence alignment of PomA/MotA and PomB/MotB, respectively, of E. coli, V. alginolyticus and B. subtilis against fully sequenced bacterial genomes by BLASTP, with an $E$-value cutoff of $<1 \times 10^{-10}$. Only pairs of directly neighbouring orthologues to $\mathrm{A}$ - and $\mathrm{B}$-subunits were considered to form a distinct stator locus. It is conceivable that not all stator complexes identified may be active.

$\ddagger$ Species comprises different isolates or subspecies exhibiting the same number of putative stator complexes.

fixed rotor-stator configurations? Is only one set of stators active at a time? Can the stators be switched? Recent studies on the bacterial rotor-stator complex suggest that stator selection may be rather dynamic.

\section{Dynamics in rotor-stator interactions}

With regard to the tight binding of the stators to the cell wall to create torque sufficient to support rotation speeds as high as $1700 \mathrm{~Hz}$ for the $\mathrm{Na}^{+}$-dependent polar flagellum 
of $V$. alginolyticus (Magariyama et al., 1994), it should be assumed that the stator ring system forms a stable and rigid complex. However, several studies strongly indicate that bacterial rotor-stator systems are surprisingly dynamic. When stator complexes were produced from an inducible promoter in an E. coli mot $A B$ mutant, a stepwise increase in rotation speed could be observed. This 'resurrection' revealed that a single stator complex is sufficient to drive flagellar rotation and demonstrated that additional stator complexes can be added to the running flagellar motor without interrupting the function. In addition, rotating motors exhibit stepwise drops and subsequent restoration of the rotation speed. These variations in rotation speed could be explained by replacement of stator complexes in the stator ring, indicating that stators can be added, but also be removed, from the running motor (Ryu et al., 2000; Sowa et al., 2005; Block \& Berg, 1984; Blair \& Berg, 1988). Direct evidence for the dynamics of rotor-stator interactions was recently presented by studies on the flagellar motor of E. coli using fluorescently labelled MotB stator components. Single stator complexes were observed to associate with, and dissociate from, the motor with a turnover time of about $30 \mathrm{~s}$ while the flagellar rotation continued (Leake et al., 2006). This exchange is assumed to occur between a pool of inactive stator precomplexes that are assembled upon expression of MotA/PomA and MotB/ PomB and diffuse freely in the cytoplasmic membrane with stator complexes associated with the flagellar motor (Fukuoka et al., 2005; Van Way et al., 2000). The precomplexes are thought to be activated upon recruitment to the flagellar motor by a conformational change that results in tight binding to the peptidoglycan and enables the flow of the coupling ion (Hosking \& Manson, 2008; Kojima et al., 2009) (Fig. 3). The reason for the high turnover rate of stators is not clear. It may prevent a decrease in motor function due to, for example, 'wearout' of the stator complexes, but this remains speculative.

A dynamic rotor-stator system, in which a membranelocated pool of stator precomplexes supports the flagellar motors, suggests intriguing possibilities with regard to stator selection. In species that possess alternative stators to drive a single flagellar motor, a dynamic exchange might allow an 'in-flight swapping' of the different stator complexes without the requirement for a completely new motor assembly. But how is the appropriate stator system selected?

Stator selection may be modulated by a combination of two major factors: (i) abundance of the stator systems, and (a)

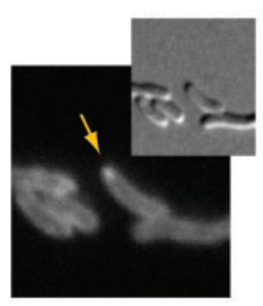

(c)

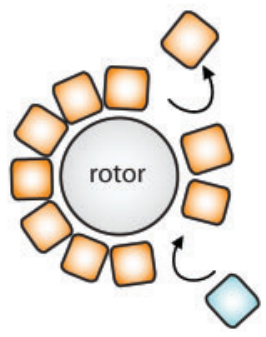

(b)

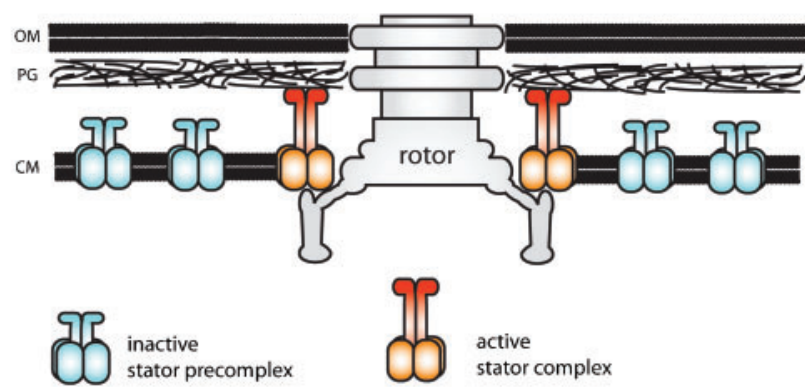

(d)

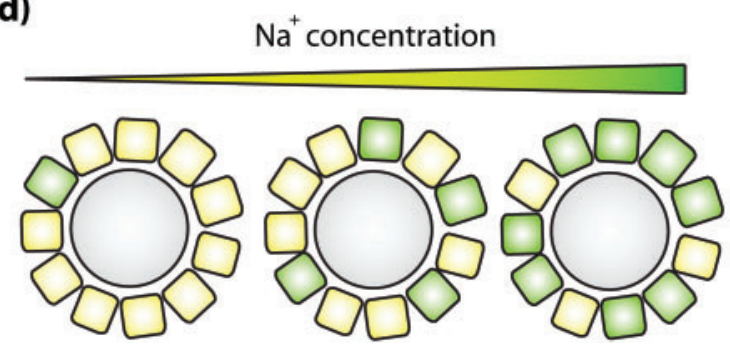

Fig. 3. Dynamic assembly of stators into the motor. (a) Micrograph of $S$. oneidensis MR-1 cells expressing mCherry-tagged PomB. The stator precomplexes are distributed in the cell envelope, and active complexes presumably cluster in the motor at the flagellated cell pole (arrow). (b, c) Active stators (red) are constantly exchanged with a membrane-embedded pool (blue). (d) Model for stator selection in S. oneidensis MR-1 in response to changes in $\mathrm{Na}^{+}$concentration. At high $\mathrm{Na}^{+}$concentrations, the PomAB stator (green) has a high incorporation efficiency and is preferentially recruited to the flagellar motor. With decreasing $\mathrm{Na}^{+}$concentration, the recruitment efficiency of the PomAB stator declines. Decreased efficiency of PomAB incorporation allows assembly of the proton-dependent MotAB stator (yellow) into the motor. 
(ii) efficiency of incorporation. Abundance-tuning of the stator precomplexes might occur at either the transcriptional or post-transcriptional level. An example of the former scenario has been described in B. subtilis, in which the genes encoding the $\mathrm{Na}^{+}$-dependent MotPS stator are thought to be transcriptionally coupled to the central reporter of carbon metabolism, CcpA. Thus, abundance of MotPS is thought be co-regulated at the transcriptional level with $\operatorname{ccp} A$ in response to cellular shifts in carbon flux (Terahara et al., 2006).

How could the incorporation efficiency into the stator ring be regulated? Recent studies on Vibrio and Shewanella species have provided insights into the stator selection process at the level of motor-stator interactions.

\section{A model for $\mathrm{Na}^{+}$-dependent stator swapping}

Rotation of the single polar flagellum of $V$. alginolyticus is driven by the $\mathrm{Na}^{+}$-dependent PomAB stators (Yorimitsu \& Homma, 2001). Fukuoka and colleagues used fluorescent fusions to the A and B stator subunits to study the dynamics of motor-stator interactions in response to environmental $\mathrm{Na}^{+}$levels. It was demonstrated that PomAB localization to the flagellated pole depends on the $\mathrm{Na}^{+}$concentration in the medium and sharply decreases in an environment containing less than $100 \mathrm{mM} \mathrm{Na}^{+}$. Furthermore, blocking $\mathrm{Na}^{+}$ transport, collapsing the $\mathrm{Na}^{+}$membrane gradient or mutating functionally critical amino acid residues resulted in delocalization of the PomAB stators (Fukuoka et al., 2009). This study demonstrated for the first time that stator localization can directly depend on the presence and concentration of its corresponding coupling ion, although the mechanism that alters interaction between motor and stator remains to be elucidated. Thus, in $V$. alginolyticus, the efficiency of motor-stator interactions is directly linked to an environmental condition, the $\mathrm{Na}^{+}$concentration in the medium. This concept can be expanded to species with two distinct stator complexes but only a single flagellar system: one stator is predominantly recruited to the flagellar motor under one set of conditions but is replaced by a second stator when the conditions change, for example, shifts in the environmental levels of $\mathrm{Na}^{+}$occur. Interestingly, this concept is in agreement with recent findings on the dualmotor system of S. oneidensis MR-1 (Paulick et al., 2009).

In S. oneidensis, either the $\mathrm{Na}^{+}$-dependent PomAB or the proton-dependent MotAB stator is sufficient to drive rotation of the single polar flagellum (Paulick et al., 2009). Localization studies on cells expressing fluorescently tagged PomB or MotB subunits revealed that both stators are simultaneously expressed and form a mixed pool of precomplexes in the cytoplasmic membrane. Under conditions of high $\mathrm{Na}^{+}$, PomAB is more efficiently incorporated into the flagellar motor and prevents recruitment of MotAB. In response to decreasing concentrations of $\mathrm{Na}^{+}$, Pom $A B$ is less efficiently recruited to the flagellar motor, and vacant positions in the stator ring can then be occupied by MotAB stator complexes (Fig. 3). This study strongly supports the model of dynamic stator swapping and, in addition, raises the possibility that rotor-stator configurations in the flagellar motor may include concurrent incorporation of different stator systems under appropriate conditions. In the example of $S$. oneidensis MR-1, this observation would suggest that the flagellum is driven by a Pom $\mathrm{AB} / \mathrm{Mot} \mathrm{AB}$ hybrid motor that could use protons and $\mathrm{Na}^{+}$ions simultaneously and whose composition is tuned by stator localization in response to $\mathrm{Na}^{+}$ concentrations (Fig. 3).

\section{Conclusions and future perspectives}

Recent findings on bacterial flagellar systems have led to a novel view about how the flagellar motor assembles. It appears that the rotor-stator complex is highly dynamic and allows functional interactions with different stator units to modify motor function. Thus, dual or multiple stator systems provide a convenient means for numerous bacterial species to tune their flagellar motors according to environmental conditions. The dynamics of rotor-stator interactions could allow dynamic stator swapping in response to external or internal cues and result in the formation of hybrid flagellar motors.

However, these exciting models are thus far based on only a few studies with a very limited number of systems. Several fundamental questions still remain unanswered. What determines the specificity of rotor-stator interactions? Do concentrations of the coupling ion, load, and/or performance of the individual stator complexes play a critical role? Is the acquisition of the appropriate stator supported by, or dependent on, additional factors, such as the auxiliary motor proteins MotX and MotY of $V$. alginolyticus (Terashima et al., 2006)? Do hybrid motors that function with different coupling ions exist? Further studies on dual and multiple stator systems of different bacterial species are required, to provide more insight into the function and the dynamics of these systems and to clarify the role they might have in functional adaptation of flagellar motors.

\section{Acknowledgements}

We thank Penelope Higgs, Sonja-Verena Albers and Chris van der Does for critically reading this manuscript and helpful discussions. We are also grateful to an anonymous reviewer whose expert comments helped to significantly improve the manuscript. We would like to thank the authors of many important works of primary research in the area of flagellar motor functions that, due to space limitations, could not be cited individually. This work was supported by the Max-Planck-Gesellschaft (MPG).

\section{References}

Armitage, J. P. (1999). Bacterial tactic responses. Adv Microb Physiol 41, 229-289.

Asai, Y., Yakushi, T., Kawagishi, I. \& Homma, M. (2003). Ioncoupling determinants of $\mathrm{Na}^{+}$-driven and $\mathrm{H}^{+}$-driven flagellar motors. J Mol Biol 327, 453-463. 
Berg, H. C. (2003). The rotary motor of bacterial flagella. Annu Rev Biochem 72, 19-54.

Blair, D. F. \& Berg, H. C. (1988). Restoration of torque in defective flagellar motors. Science 242, 1678-1681.

Block, S. M. \& Berg, H. C. (1984). Successive incorporation of forcegenerating units in the bacterial rotary motor. Nature 309, 470-472.

Braun, T. F., Al-Mawsawi, L. Q., Kojima, S. \& Blair, D. F. (2004). Arrangement of core membrane segments in the MotA/MotB protonchannel complex of Escherichia coli. Biochemistry 43, 35-45.

Coulton, J. W. \& Murray, R. G. (1978). Cell envelope associations of Aquaspirillum serpens flagella. J Bacteriol 136, 1037-1049.

Dobell, C. (1960). Antony van Leeuwenhoek and His "Little Animals". New York: Dover.

Doyle, T. B., Hawkins, A. C. \& McCarter, L. L. (2004). The complex flagellar torque generator of Pseudomonas aeruginosa. J Bacteriol 186, 6341-6350.

Fukuoka, H., Yakushi, T., Kusumoto, A. \& Homma, M. (2005). Assembly of motor proteins, PomA and PomB, in the $\mathrm{Na}^{+}$-driven stator of the flagellar motor. J Mol Biol 351, 707-717.

Fukuoka, H., Wada, T., Kojima, S., Ishijima, A. \& Homma, M. (2009). Sodium-dependent dynamic assembly of membrane complexes in sodium-driven flagellar motors. Mol Microbiol 71, 825-835.

Gosink, K. K. \& Häse, C. C. (2000). Requirements for conversion of the $\mathrm{Na}^{+}$-driven flagellar motor of Vibrio cholerae to the $\mathrm{H}^{+}$-driven motor of Escherichia coli. J Bacteriol 182, 4234-4240.

Hosking, E. R. \& Manson, M. D. (2008). Clusters of charged residues at the $\mathrm{C}$ terminus of MotA and $\mathrm{N}$ terminus of MotB are important for function of the Escherichia coli flagellar motor. J Bacteriol 190, 55175521.

Ito, M., Hicks, D. B., Henkin, T. M., Guffanti, A. A., Powers, B. D., Zvi, L., Uematsu, K. \& Krulwich, T. A. (2004). MotPS is the statorforce generator for motility of alkaliphilic Bacillus, and its homologue is a second functional Mot in Bacillus subtilis. Mol Microbiol 53, 10351049.

Ito, M., Terahara, N., Fujinami, S. \& Krulwich, T. A. (2005). Properties of motility in Bacillus subtilis powered by the $\mathrm{H}^{+}$-coupled MotAB flagellar stator, $\mathrm{Na}^{+}$-coupled MotPS or hybrid stators MotAS or MotPB. J Mol Biol 352, 396-408.

Khan, S., Dapice, M. \& Reese, T. S. (1988). Effects of mot gene expression on the structure of the flagellar motor. J Mol Biol 202, 575584 .

Khan, S., Ivey, D. M. \& Krulwich, T. A. (1992). Membrane ultrastructure of alkaliphilic Bacillus species studied by rapid-freeze electron microscopy. J Bacteriol 174, 5123-5126.

Koerdt, A., Paulick, A., Mock, M., Jost, K. \& Thormann, K. M. (2009). MotX and MotY are required for flagellar rotation in Shewanella oneidensis MR-1. J Bacteriol 191, 5085-5093.

Kojima, S. \& Blair, D. F. (2004a). The bacterial flagellar motor: structure and function of a complex molecular machine. Int Rev Cytol 233, 93-134.

Kojima, S. \& Blair, D. F. (2004b). Solubilization and purification of the MotA/MotB complex of Escherichia coli. Biochemistry 43, 26-34.

Kojima, S., Shinohara, A., Terashima, H., Yakushi, T., Sakuma, M., Homma, M., Namba, K. \& Imada, K. (2008). Insights into the stator assembly of the Vibrio flagellar motor from the crystal structure of MotY. Proc Natl Acad Sci U S A 105, 7696-7701.

Kojima, S., Imada, K., Sakuma, M., Sudo, Y., Kojima, C., Minamino, T., Homma, M. \& Namba, K. (2009). Stator assembly and activation mechanism of the flagellar motor by the periplasmic region of MotB. Mol Microbiol 73, 710-718.
Leake, M. C., Chandler, J. H., Wadhams, G. H., Bai, F., Berry, R. M. \& Armitage, J. P. (2006). Stoichiometry and turnover in single, functioning membrane protein complexes. Nature 443, 355-358.

Liu, J., Lin, T., Botkin, D. J., McCrum, E., Winkler, H. \& Norris, S. J. (2009). Intact flagellar motor of Borrelia burgdorferi revealed by cryoelectron tomography: evidence for stator ring curvature and rotor/ C-ring assembly flexion. J Bacteriol 191, 5026-5036.

Lloyd, S. A. \& Blair, D. F. (1997). Charged residues of the rotor protein FliG essential for torque generation in the flagellar motor of Escherichia coli. J Mol Biol 266, 733-744.

Lloyd, S. A., Tang, H., Wang, X., Billings, S. \& Blair, D. F. (1996). Torque generation in the flagellar motor of Escherichia coli: evidence of a direct role for FliG but not for FliM or FliN. J Bacteriol 178, 223231.

Macnab, R. M. (2003). How bacteria assemble flagella. Annu Rev Microbiol 57, 77-100.

Magariyama, Y., Sugiyama, S., Muramoto, K., Maekawa, Y., Kawagishi, I., Imae, Y. \& Kudo, S. (1994). Very fast flagellar rotation. Nature 371, 752.

McCarter, L. L. (2006). Regulation of flagella. Curr Opin Microbiol 9, 180-186.

McCarter, L., Hilmen, M. \& Silverman, M. (1988). Flagellar dynamometer controls swarmer cell differentiation of Vibrio parahaemolyticus. Cell 54, 345-351.

Merino, S., Shaw, J. G. \& Tomás, J. M. (2006). Bacterial lateral flagella: an inducible flagella system. FEMS Microbiol Lett 263, 127-135.

Minamino, T., Imada, K. \& Namba, K. (2008). Molecular motors of the bacterial flagella. Curr Opin Struct Biol 18, 693-701.

Paulick, A., Koerdt, A., Lassak, J., Huntley, S., Wilms, I., Narberhaus, F. \& Thormann, K. M. (2009). Two different stator systems drive a single polar flagellum in Shewanella oneidensis MR-1. Mol Microbiol 71, 836850.

Reid, S. W., Leake, M. C., Chandler, J. H., Lo, C. J., Armitage, J. P. \& Berry, R. M. (2006). The maximum number of torque-generating units in the flagellar motor of Escherichia coli is at least 11. Proc Natl Acad Sci U S A 103, 8066-8071.

Ryu, W. S., Berry, R. M. \& Berg, H. C. (2000). Torque-generating units of the flagellar motor of Escherichia coli have a high duty ratio. Nature 403, 444-447.

Sato, K. \& Homma, M. (2000). Functional reconstitution of the $\mathrm{Na}^{+}$-driven polar flagellar motor component of Vibrio alginolyticus. J Biol Chem 275, 5718-5722.

Shimada, T., Sakazaki, R. \& Suzuki, K. (1985). Peritrichous flagella in mesophilic strains of Aeromonas. Jpn J Med Sci Biol 38, 141-145.

Sowa, Y. \& Berry, R. M. (2008). Bacterial flagellar motor. Q Rev Biophys 41, 103-132.

Sowa, Y., Rowe, A. D., Leake, M. C., Yakushi, T., Homma, M., Ishijima, A. \& Berry, R. M. (2005). Direct observation of steps in rotation of the bacterial flagellar motor. Nature 437, 916-919.

Terahara, N., Fujisawa, M., Powers, B., Henkin, T. M., Krulwich, T. A. \& Ito, M. (2006). An intergenic stem-loop mutation in the Bacillus subtilis ccpA-motPS operon increases motPS transcription and the MotPS contribution to motility. J Bacteriol 188, 2701-2705.

Terashima, H., Fukuoka, H., Yakushi, T., Kojima, S. \& Homma, M. (2006). The Vibrio motor proteins, MotX and MotY, are associated with the basal body of $\mathrm{Na}^{+}$-driven flagella and required for stator formation. Mol Microbiol 62, 1170-1180.

Thomas, D. R., Francis, N. R., Xu, C. \& DeRosier, D. J. (2006). The three-dimensional structure of the flagellar rotor from a clockwiselocked mutant of Salmonella enterica serovar Typhimurium. J Bacteriol 188, 7039-7048. 
Toutain, C. M., Zegans, M. E. \& O'Toole, G. A. (2005). Evidence for two flagellar stators and their role in the motility of Pseudomonas aeruginosa. J Bacteriol 187, 771-777.

Toutain, C. M., Caizza, N. C., Zegans, M. E. \& O'Toole, G. A. (2007). Roles for flagellar stators in biofilm formation by Pseudomonas aeruginosa. Res Microbiol 158, 471-477.

Van Way, S. M., Hosking, E. R., Braun, T. F. \& Manson, M. D. (2000). Mot protein assembly into the bacterial flagellum: a model based on mutational analysis of the motB gene. J Mol Biol 297, 7-24.

Wang, Q., Suzuki, A., Mariconda, S., Porwollik, S. \& Harshey, R. M. (2005). Sensing wetness: a new role for the bacterial flagellum. EMBO J 24, 2034-2042.

Wilhelms, M., Vilches, S., Molero, R., Shaw, J. G., Tomas, J. M. \& Merino, S. (2009). Two redundant sodium-driven stator motor proteins are involved in Aeromonas hydrophila polar flagellum rotation. J Bacteriol 191, 2206-2217.

Yakushi, T., Yang, J., Fukuoka, H., Homma, M. \& Blair, D. F. (2006).

Roles of charged residues of rotor and stator in flagellar rotation: comparative study using $\mathrm{H}^{+}$-driven and $\mathrm{Na}^{+}$-driven motors in Escherichia coli. J Bacteriol 188, 1466-1472.

Yorimitsu, T. \& Homma, M. (2001). $\mathrm{Na}^{+}$-driven flagellar motor of Vibrio. Biochim Biophys Acta 1505, 82-93.

Yorimitsu, T., Sowa, Y., Ishijima, A., Yakushi, T. \& Homma, M. (2002).

The systematic substitutions around the conserved charged residues of the cytoplasmic loop of $\mathrm{Na}^{+}$-driven flagellar motor component PomA. J Mol Biol 320, 403-413.

Yorimitsu, T., Mimaki, A., Yakushi, T. \& Homma, M. (2003). The conserved charged residues of the C-terminal region of FliG, a rotor component of the $\mathrm{Na}^{+}$-driven flagellar motor. J Mol Biol 334, 567583.

Zhou, J. \& Blair, D. F. (1997). Residues of the cytoplasmic domain of MotA essential for torque generation in the bacterial flagellar motor. J Mol Biol 273, 428-439.

Zhou, J., Lloyd, S. A. \& Blair, D. F. (1998). Electrostatic interactions between rotor and stator in the bacterial flagellar motor. Proc Natl Acad Sci U S A 95, 6436-6441. 